\title{
Etude expérimentale de l'action cancérigène du radon 222 Comparaison avec les études épidémiologiques
}

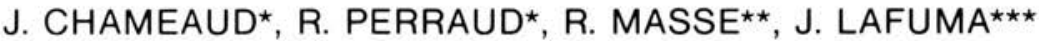

\begin{abstract}
RÉSUMÉ
Depuis plus de dix ans, des expériences ont été pratiquées au CEA pour mesurer, chez le rat, l'action cancérigène des produits de filiation du radon 222. Les résultats obtenus permettent de définir une relation dose-effet pour des doses comprises entre 60 et 8000 WLM (1). Ces résultats sont comparables à ceux fournis par les enquêtes épidémiologiques pratiquées sur diverses populations de mineurs d'uranium.
\end{abstract}

On analyse, de plus, l'action de paramètres tels que le débit de dose, l'étalement de la dose et l'âge des animaux au moment de l'irradiation. Enfin, on étudie l'action de divers cofacteurs dont la fumée de cigarettes. Les résultats montrent que l'expérimentation animale permettra d'extrapoler les effets chez l'homme pour des doses plus faibles que celles pour lesquelles on possède déjà des résultats d'enquêtes épidémiologiques.

\section{ABSTRACT}

For over ten years, investigations have been carried out at the French Commissariat à l'Energie Atomique in order to measure in the rat the carcinogenic action of radon 222 daughter products. The results allow to define a dose-effect relationship for doses ranging from 60 to 8000 WLM (1). These results can be compared with those supplied by epidemiological surveys conducted on various populations of uranium miners.

The effects of various parameters such as dose-rate, dose protraction and age of the animals at exposure are also analyzed. At last, the effects of various co-factors including cigarette smoke are studied. The results show that animal investigation makes it possible to extrapolate to man for doses lower than those for which results of epidemiological surveys are available.

* CogemA, Division de la Crouzille, B.P. n॰1, 87640 Razes.

** Commissariat à l'énergie atomique, IPSN, Département de protection, Centre d'études de Bruyères-le-Chatel (CEB III), B.P. $n^{\circ} 561,92120$ Montrouge.

*** Commissariat à l'énergie atomique, IPSN, Département de Protection, B.P. $n^{\circ} 6$, 92260 Fontenay-aux-Roses.

(1) WLM (working level month) : 1 WLM équivaut à 3,6 millijoules par mètre cube par heure. 
Les risques de cancer pulmonaire après inhalation de radon sont estimés aujourd'hui, chez l'homme, en utilisant deux approches : épidémiologiques et dosimétriques.

L'épidémiologie a tiré ses données des enquêtes pratiquées chez les mineurs d'uranium et l'approche dosimétrique utilise des calculs théoriques basés sur des modèles prenant en compte aussi bien les caractéristiques des aérosols que celles du poumon humain. Bien que les résultats des deux approches soient voisins, un certain nombre de points restent controversés.

La complexité et l'instabilité des aérosols présents dans l'atmosphère de la mine et la difficulté pour le modèle le plus sophistiqué de reproduire exactement le poumon humain font peser une incertitude quant à la dose exacte délivrée aux différentes parties de l'appareil respiratoire. Le fait que l'on ne connaisse pas aujourd'hui les mécanismes impliqués dans la génèse des cancers ne permet pas de savoir quelles sont les cellules cibles, donc d'apprécier pleinement le rôle de la distribution topographique de l'irradiation.

Les enquêtes épidémiologiques d'un autre côté, en dépit de la qualité des études, souffrent des incertitudes qui existent sur les doses réellement absorbées par les différents individus. En outre, les informations que l'on possède aujourd'hui proviennent d'expositions anciennes dont les niveaux sont supérieurs à ceux rencontrés actuellement. II est donc nécessaire d'extrapoler avec toutes les incertitudes que celà comporte. Enfin, l'épidémiologie ne permet pas de dissocier l'action du radon de celle des autres polluants présents dans l'atmosphère minière, ni d'apprécier avec précision le rôle de l'action synergique de la fumée de cigarettes.

L'expérimentation animale a été largement pratiquée pour évaluer la toxicité de divers radionucléides pour lesquels on ne dispose pas de données humaines. Il est surprenant de constater le faible nombre d'expériences consacrées à l'action du radon 222. Ceci est d'autant plus curieux que l'on est en présence d'un des rares cas pour lequel une comparaison des résultats acquis sur l'homme et sur l'animal peut être aisément pratiquée.

Depuis quinze ans nous avons pratiqué de nombreuses expériences couvrant une large gamme de doses et bien que les deux dernières ne soient pas encore terminées, il est possible aujourd'hui de comparer l'homme et l'animal pour les doses pour lesquelles existent des données humaines. Aux plus faibles doses, en dessous de 50 WLM, les données expérimentales offrent une possibilité sérieuse de vérifier la validité des extrapolations à partir des fortes doses humaines, car il n'existe pas aujourd'hui de données humaines à faibles doses.

\section{MATÉRIEL ET MÉTHODES}

Toutes les expériences ont été pratiquées sur des rats mâles SpragueDawley âgés d'environ 3 mois au début des inhalations. La taille réduite de ces rongeurs permet d'en utiliser simultanément un grand nombre. Leur durée de vie, suffisante pour apprécier les différences de temps de latence, est assez courte pour que la durée de l'expérience -trois ans- soit raisonnable. Le taux d'apparition spontanée de cancers pulmonaires est faible dans cette souche [8]. Enfin, les rats, contrairement aux souris, sont indemnes de virus transmis d'une génération à l'autre et qui, dans certains cas, entraînent l'apparition d'un taux élevé de tumeurs pulmonaires. 


\section{Inhalation de radon et de ses produits de filiation}

Les dispositifs d'inhalation ont été déjà décrits [2]. Le premier dispositif comprenait une chambre de $0,5 \mathrm{~m}^{3}$ associée à un générateur renfermant du minerai d'uranium très riche. Plus tard, une nouvelle installation comportant une chambre de $10 \mathrm{~m}^{3}$ fut construite. Elle permet d'utiliser simultanément jusqu'à 500 rats et de leur faire inhaler des produits de filiation du radon, à des concentrations allant de 100 à $10000 \mathrm{WL}$ pendant des temps variables (1 à $10 \mathrm{~h} / \mathrm{j})$. Les concentrations sont plus élevées que celles rencontrées dans les mines d'uranium, mais c'est le seul moyen pour faire inhaler à un rat la même dose totale que celle inhalée par l'homme pendant la même fraction de leur durée de vie respective.

Les concentrations en radon et l'état d'équilibre des produits de filiation sont soigneusement contrôlés à chaque séance.

\section{Inhalation de poussières}

Le dispositif permet d'utiliser simultanément 20 à 30 animaux dans une chambre d'inhalation dans laquelle la concentration en poussière est maintenue constante [7].

\section{Inhalation de fumées de cigarettes}

On a utilisé, pour faire inhaler aux rats de la fumée de cigarettes, une chambre étanche de $0,5 \mathrm{~m}^{3}$ contenant jusqu'à 50 animaux. La concentration est égale à la quantité de fumée produite par 18 cigarettes et répartie dans $1 \mathrm{~m}^{3}$. Les séances d'inhalation sont de $i 0 \mathrm{~min}$ et répétées plusieurs fois par jour [3].

\section{Analyses histologiques}

Les rats sont analysés soit après leur mort spontanée, soit après avoir été sacrifiés moribonds.

Les poumons sont prélevés en entier et fixés suivant un protocole bien défini, puis ils sont coupés en tranches de $20 \mu \mathrm{m}$. Si une anomalie est décelée, on prélève un échantillon de $5 \mu \mathrm{m}$ d'épaisseur. Après coloration, l'échantillon est analysé et la lésion définie suivant la classification utilisée dans le laboratoire [5].

Plus de 10000 animaux ont été utilisés dans ces différentes recherches et plus de 600 cancers du poumon ont été observés.

\section{RÉSULTATS}

\section{Lésions pulmonaires observées}

Ces lésions sont soit bénignes (pneumonie interstitielle, adénomatose, métaplasie, adénomes et papillomes), soit malignes. Nous n'analyserons que les lésions cancéreuses.

Le cancer pulmonaire du rat a la réputation d'être très différent de celui de l'homme. Mais ce point mérite d'être discuté. Nous avons revu récemment 500 cancers pulmonaires de rats qui ont été classés selon les règles de l'OMS après consultation de pathologistes humains. Le tableau I résume les résultats. Seuls, deux types sont peu représentés chez l'homme : les carcinomes 
alvéolaires et bronchiolo-alvéolaires. Par contre, les autres types qui proviennent des cellules de l'arbre bronchique sont très comparables chez le rat et chez l'homme. Le cancer anaplasique à petites cellules (oat-cells) qui représente une certaine fraction des cancers humains, n'existe pas chez le rat.

TABLEAU I

Cancers pulmonaires chez le rat

\begin{tabular}{|c|c|c|}
\hline & $\mathrm{Nb}$ & $\%$ \\
\hline $\begin{array}{l}\text { Carcinomes : } 498 \\
\text { - épidermoïdes } \\
\text { - adénocarcinomes } \\
\text { - anaplasiques : } \\
\quad \text { cellules géantes } \\
\quad \text { petites cellules } \\
\text { - bronchiolo-alvéolaires } \\
\text { - alvéolaires } \\
\text { Sarcomes : } 2 \\
\text { - angiosarcomes }\end{array}$ & $\begin{array}{r}262 \\
75 \\
\\
11 \\
0 \\
139 \\
11 \\
2\end{array}$ & $\begin{array}{c}56,4 \\
15,0 \\
\\
2,2 \\
0 \\
27,8 \\
2,2 \\
\end{array}$ \\
\hline
\end{tabular}

En plus des cancers pulmonaires, il n'y a que deux autres cancers pour lesquels un excès a été constaté chez les rats inhalant de fortes doses de radon. Ce sont, d'une part, les épithéliomas cutanés de la lèvre supérieure et, d'autre part les cancers de l'appareil urinaire. Cette dernière localisation est due au fait que la dose absorbée au niveau du rein et provenant de la dispersion dans l'organisme des produits de filiation de radon inhalés suffit à induire ces cancers, étant donné la grande sensibilité du rein des rats SpragueDawley.

\section{Le temps de latence}

Nous appelons temps de latence, l'intervalle de temps qui s'écoule entre le début des inhalations et le jour où la lésion cancéreuse est constatée (à l'autopsie de l'animal). Le temps de latence dépend de plusieurs facteurs :

\subsection{Facteurs physiques - la dose cumulée}

Le tableau II résume les résultats et montre que plus la dose est élevée, plus le temps de latence est court. Si ce fait existe chez l'homme, comme l'a suggéré ARCHER [1], il en résulte que, dans une cohorte, l'excès des cancers sera décelé d'autant plus tardivement que la dose aura été plus faible.

\subsection{Facteurs biologiques}

Rôle de l'âge au moment de l'exposition. Le tableau III montre que pour une même dose, plus l'âge est élevé au début des inhalations et plus le temps de latence est court. Ce fait a également été observé chez les mineurs d'uranium [10]. 
TABLEAU II

Latence des cancers pulmonaires du rat

\begin{tabular}{|c|c|}
\hline Dose (WLM) & Latence (jours) \\
\hline 65 & 712 \\
170 & 748 \\
290 & 742 \\
860 & 768 \\
1470 & 690 \\
3000 & 714 \\
3800 & 575 \\
3900 & 652 \\
4500 & 659 \\
6000 & 658 \\
6000 & 648 \\
8000 & 613 \\
\hline
\end{tabular}

TABLEAU III

Effet de l'àge au début des inhalations (6000 WLM)

\begin{tabular}{|c|c|}
\hline Age (jours) & Latence (jours) \\
\hline 150 & 640 \\
280 & 510 \\
400 & 450 \\
520 & 305 \\
\hline
\end{tabular}

Sensibilité individuelle. Dans la plupart des expériences, on voit apparaître très rapidement un ou deux cancers pulmonaires très invasifs et ce, quelle que soit la dose. Ce fait traduit l'existence d'une sensibilité individuelle très élevée malgré l'homogénéité de la souche utilisée.

\section{Fréquence}

La fréquence croît avec la dose cumulée. Aux faibles doses, une relation linéaire ne peut être exclue. La fréquence dépend, en plus de la dose cumulée, de plusieurs autres paramètres :

\subsection{La durée de vie des animaux}

Aux très fortes doses, la durée de vie des rats est plus raccourcie que ne l'est le temps de latence; aussi la fréquence des cancers observés estelle plus faible qu'elle ne devrait l'être.

Quand les animaux sont soumis aux inhalations à un âge avancé, la fréquence des cancers observés est diminuée par le fait que certains animaux meurent avant d'avoir exprimé leur cancer.

\subsection{Les parametres physiques}

Le débit de dose. Le tableau IV montre qu'à dose cumulée égale, l'élévation du débit de dose diminue la fréquence des cancers. Ce fait a également été retrouvé chez l'homme par SEVC [9].

\section{TABLEAU IV}

Effet du débit de dose

\begin{tabular}{|c|c|}
\hline \multicolumn{2}{|c|}{ Faibles débits de dose (Concentration < $3000 \mathrm{WL}$ ) } \\
Dose & $\%$ de cancers \\
1500 & 25 \\
3000 & 42 \\
3900 & 34 \\
6000 & 43 \\
\hline Débits de doses élevés (Concentration $=12000 \mathrm{WL})$ \\
Dose & $\%$ de cancers \\
2250 & 28 \\
3700 & 36 \\
5400 & 24 \\
7000 & 32 \\
\hline
\end{tabular}


Le fractionnement de la dose. Pour une même dose cumulée, et pour une même concentration atmosphérique, le fractionnement de la dose accroît l'incidence des cancers (tableau V).

\section{TABLEAU $V$}

Effet du fractionnement de la dose. (Dose totale : 6000 WLM ; concentration : $3000 \mathrm{WL}$; durée des inhalations : $7 \mathrm{~h} / \mathrm{j}$ )

\begin{tabular}{|c|c|c|}
\hline Nb de jours/semaine & Nb de rats & Nb de cancers pulmonaires \\
\hline 5 & 75 & 17 \\
1 & 40 & 35 \\
\hline
\end{tabular}

\section{Forme de la relation dose-effet}

Le tableau VI résume les données expérimentales de ces dernières années. II montre que l'incidence par unité de dose croît quand la dose totale diminue. La relation dose-effet est vraisemblablement à concavité inférieure entre 65 et 8000 WLM (fig. 1).

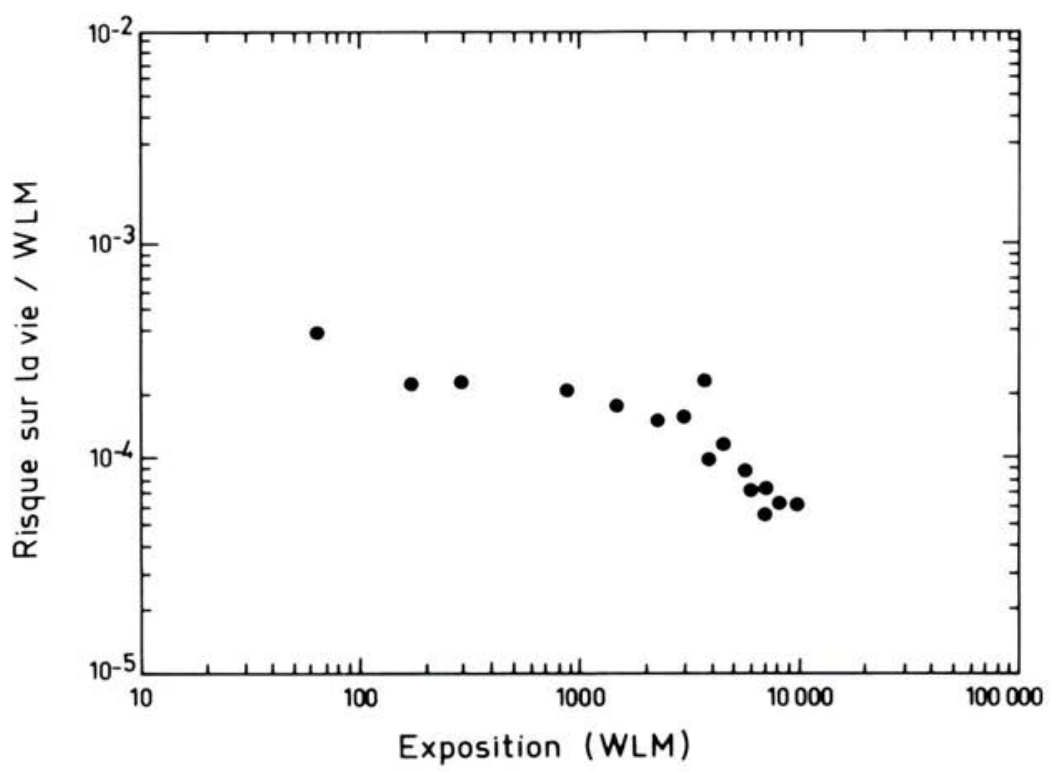

Fig. 1. - Coefficients de risque sur la vie pour l'exposition aux produits de filiation du radon. 
TABLEAU VI

Relation dose-effet

\begin{tabular}{|c|c|c|c|}
\hline $\begin{array}{c}\text { Dose } \\
(\text { WLM })\end{array}$ & $\begin{array}{c}\text { Rats } \\
\text { (Nb) }\end{array}$ & $\begin{array}{c}\text { Cancers pulmonaires } \\
(\mathrm{Nb})\end{array}$ & $\begin{array}{c}\text { Incidence } \\
\left(10^{-6} \text { par WLM }\right)\end{array}$ \\
\hline 65 & 500 & 12 & 370 \\
170 & 294 & 14 & 276 \\
290 & 21 & 2 & 328 \\
860 & 20 & 4 & 233 \\
1470 & 20 & 5 & 170 \\
3000 & 40 & 17 & 142 \\
3800 & 20 & 7 & 92 \\
3900 & 50 & 17 & 87 \\
4500 & 40 & 29 & 161 \\
6000 & 29 & 12 & 69 \\
6000 & 25 & 11 & 73 \\
8000 & 180 & 76 & 53 \\
\hline
\end{tabular}

Etant donné l'imprécision sur les fréquences aux faibles doses, on peut faire passer par ces points une relation linéaire sans seuil.

Nous avons, en 1981, lancé trois nouvelles séries expérimentales de 500 rats chacunes, à 0, 20 et 40 WLM pour explorer la gamme des faibles doses.

\section{ROLE DES COFACTEURS}

Nous avons recherché l'action de deux types de cofacteurs: ceux qui sont naturellement présents dans l'atmosphère des mines d'uranium et la fumée de cigarettes.

\section{Autres polluants présents dans l'atmosphère des mines d'uranium}

Trois d'entre eux ont été étudiés: la poussière de silice, la poussière de minerai d'uranium et les suies produites par les moteurs Diesel.

\subsection{Silice}

Les études ont été menées dans le but de savoir si la présence de radon aggraverait le développement du processus silicotique. Les expériences ont permis d'établir que cette synergie n'existait pas, ce qui est confirmé par le fait que chez les mineurs d'uranium, les silicoses ne sont ni plus fréquentes ni plus rapidement évolutives que chez les autres mineurs.

Les doses de silice utilisées ont tellement raccourci la vie des animaux qu'il n'a pas été observé de cancers pulmonaires.

\subsection{Poussières de minerai}

Dans ces expériences, les rats ont inhalé de fortes doses de radon associées ou non à de fortes concentrations en minerai d'uranium très riche. Le nombre d'animaux était trop faible pour que l'absence de différence constatée ait été significative. Un résultat similaire a été trouvé par CROSS au Pacific Northwest laboratory [4]. 


\subsection{Suies provenant des Diesel}

La toxicité chimique des produits émis par les Diesel est bien connue. Elle entraîne l'aggravation des bronchites chroniques. Mais son rôle sur l'induction des cancers pulmonaires est très discuté.

Les expériences animales pratiquées en faisant inhaler directement de l'air provenant de pots d'échappement de moteurs Diesel n'ont pas permis de mettre en évidence une éventuelle action cancérigène. Mais il est possible que la forte toxicité du produit (CO) n'ait pas permis d'administrer une dose suffisante.

Aussi, avons-nous recueilli sur filtre des échantillons de fumées d'échappement de Diesel qui, après prélèvement, ont été administrées par voie intratrachéale à des animaux ayant préalablement inhalé du radon. Aux fortes doses de radon, on a observé une tendance non significative au raccourcissement du temps de latence. Aux faibles doses de radon, on n'a pas observé de différence entre les deux lots d'animaux.

Etant données les très fortes doses utilisées, aussi bien pour le radon que pour les fumées de Diesel, il est très peu probable qu'un effet synergique entre les deux polluants puisse être observé aux faibles doses auxquelles ils sont rencontrés dans l'atmosphère des mines.

\section{Fumée de cigarettes}

Un effet synergique important a été observé entre le radon et la fumée de cigarettes. Le tableau VII résume les résultats d'expériences pratiquées à dose de fumée de cigarettes identique $(350 \mathrm{~h}$ dans les conditions standardisées du laboratoire) et à doses de radon variables. En plus de l'augmentation de fréquence, on a observé que chez les animaux qui ont, en plus du radon, inhalé de la fumée de cigarettes, les cancers pulmonaires étaient beaucoup plus précoces, plus gros, et plus invasifs.

TABLEAU VII

Effet de la fumée de cigarettes

\begin{tabular}{|c|c|c|c|}
\hline & $\begin{array}{l}\text { Dose } \\
\text { (WLM) }\end{array}$ & $\begin{array}{l}\text { Rats } \\
\text { (Nb) }\end{array}$ & $\begin{array}{c}\text { Cancers pulmonaires } \\
(\mathrm{Nb})\end{array}$ \\
\hline 4000 & {$\left[\begin{array}{l}\text { radon } \\
\text { radon }+ \text { fumée }\end{array}\right.$} & $\begin{array}{l}50 \\
50\end{array}$ & $\begin{array}{l}17 \\
34\end{array}$ \\
\hline 500 & {$\left[\begin{array}{l}\text { radon } \\
\text { radon }+ \text { fumée }\end{array}\right.$} & $\begin{array}{l}28 \\
30\end{array}$ & $\begin{array}{l}2 \\
8\end{array}$ \\
\hline 100 & {$\left[\begin{array}{l}\text { radon } \\
\text { radon + fumée }\end{array}\right.$} & $\begin{array}{l}28 \\
30\end{array}$ & $\begin{array}{l}0 \\
1\end{array}$ \\
\hline 0 & fumée & 45 & 0 \\
\hline
\end{tabular}

Dans ces expériences, la fumée de cigarettes avait été administrée après le radon. Nous avons effectué une autre expérience pour étudier le 
rôle de l'ordre d'administration des deux facteurs. Le tableau VIII résume les résultats et montre clairement que l'effet synergique n'est observé que si l'inhalation de fumée de cigarettes suit celle de radon.

TABLEAU VIII

Effet de la fumée de cigarettes (400 WLM)

\begin{tabular}{|l|c|c|}
\hline & $\begin{array}{c}\text { Rats } \\
\mathrm{Nb}\end{array}$ & $\begin{array}{c}\text { Cancers pulmonaires } \\
\mathrm{Nb}\end{array}$ \\
\hline Radon & 50 & 12 \\
Tabac avant le radon & 50 & 7 \\
Tabac après le radon & 50 & 41 \\
\hline
\end{tabular}

Actuellement, nous avons en cours une expérience dans laquelle le radon a été administré à la même dose et la fumée de cigarettes à doses variables pour étudier la double relation dose-effet.

\section{DISCUSSION}

On peut aujourd'hui comparer les résultats de nos expériences avec ceux fournis par les enquêtes épidémiologiques. II existe, entre l'homme et le rat soumis au radon, des différences et des ressemblances.

\section{Différences}

1) Pour des doses cumulées semblables, l'animal doit être exposé à des atmosphères dont les concentrations sont plus élevées que pour l'homme.

2) En expérimentation, les doses inhalées sont connues avec beaucoup plus de précision.

3) En expérimentation, les cofacteurs sont bien maîtrisés.

4) Le point de départ des cancers pulmonaires n'est pas le même chez le rat et chez l'homme.

Les différences semblent liées à l'espèce plutôt qu'à la distribution topographique des doses. En effet, chez les rongeurs et les félins, l'inhalation de radon comme celle d'oxyde de plutonium dont la rétention est alvéolaire entraîne l'apparition de cancers pulmonaires.

Chez l'homme ayant inhalé du radon, comme chez les primates ayant inhalé de l'oxyde de plutonium à rétention alvéolaire, les cancers ont un point de départ bronchique.

5) Les types histologiques des cancers sont différents chez le rat et l'homme. Notamment, le cancer anaplasique à petites cellules (oat-cells) n'existe pas chez le rat.

6) Chez le rat et l'homme, on observe l'invasion des chaînes lymphatiques. Cependant, chez le rat, on n'observe qu'exceptionnellement des métastases dans d'autres organes. 


\section{Ressemblances}

1) Dans la gamme des doses analysées, la forme de la relation dose-effet est la même. Dans les deux espèces, l'incidence par unité de dose croît quand la dose totale diminue.

2) Pour une même dose absorbée, la période de latence est plus courte quand l'âge au début des inhalations de radon est plus avancé.

3) Pour une même dose totale, l'incidence des tumeurs croît quand le débit de dose décroît.

\section{Points établis chez l'animal et discutés chez l'homme}

1) L'action synergique de la fumée de cigarettes [6].

2) La diminution de la période de latence quand la dose cumulée est plus élevée.

\section{v. CONCLUSIONS}

Malgré d'indéniables différences entre les deux espèces, les ressemblances constatées après inhalation de radon sont suffisamment importantes pour valider le modèle animal. La plus importante est la similitude de la forme des relations dose-effet. C'est pour cette raison que nous étudions actuellement l'action de doses aussi faibles que 20 et 40 WLM, car nous pensons que les résultats seront une base sérieuse pour prévoir les risques humains dans cette gamme de doses.

\section{BIBLIOGRAPHIE}

[1] ARCHER V.E., WAGONER J.K., LUNDIN F.E. Cancer mortality among uranium mill workers. J. Occup. Med., 1973, 15, 11-14.

[2] ChAMEAUD J., PERRAUD R., LAFUMA J., MASSE R. Cancers du poumon expérimentaux provoqués chez le rat par des inhalations de radon. C.R. Acad. Sci., Série D, 1971. 273. ว३RЯ-2२२ด

[3] ChAMEAUD J., PERRAUD R., CHRETIEN J., MASSE R., LAFUMA J. Combined effects of inhalation of radon daughter products and tobacco smoke. In : Pulmonary toxicology of respirable particles, 19th Annual Hanford life sciences symposium, Oct. 22-24, 1979 (Sanders, C.L., Ed.). Spingfield: NTIS, 1979, 551 - 557 (DOE Symposium Series 53).

[4] CROSS F.T., PALMER R.F., BUSCH R.H. and BUSCHBOM R.L. Influence of radon daughter exposure rate and uranium ore dust concentration on occurence of lung tumors. PNL - 3300 Pt 1, 1980, p 130.

[5] MASSE R. Histogenesis of lung tumors induced in rats by inhalation of $\alpha$-emitters, an overview. In : Pulmonary toxicology of respirable particles, 19th Annual Hanford life sciences symposium, Oct. 22-24, 1979 (Sanders, C.L., Ed.). Spingfield : NTIS, 498-520 (DOE Symposium Series 53).

[6] National Academy of Sciences. Committee on the biological effects of ionizing radiation, BEIR Report III. Washington, D.C. : National academy of sciences, 1980.

[7] PERRAUD R., CHAMEAUD J., MASSE R., LAFUMA J. Cancers pulmonaires expérimentaux chez le rat après inhalation de radon associé à des poussières non radioactives. C.R. Acad. Sci., Série D, 1970, 270, 2594-2595. 
[8] SANDERS C.L., MAHAFFEY J.A. Inhalation toxicology of transuranics in rodents. In : Biological implications of radionuclides released from nuclear industry, Vienna, 26-30 March 1979. Vienna : AIEA, 1979, 1, 89-104.

[9] SEVC J., KUNZ E., PLACEK V. Lung cancer in uranium miners and long-term exposure to radon daughters products. Health Phys. 1976, 30, 433-437.

[10] UNSCEAR. Comité scientifique des Nations Unies pour l'étude des effets des rayonnements ionisants. Rapport à l'Assemblée générale, 1977. New York: Nations Unies, 1978. 